\section{Recent Orthopedic Surgeries Aiming to Improve Quality of Life for Patients with Rheumatoid Arthritis}

\section{To the Editor:}

It was with great interest that we read the article by Zhong, et al ${ }^{1}$. They examined the rate and variation in rheumatoid arthritis (RA)-related hand and wrist surgery. They concluded that surgical reconstruction of rheumatoid hand deformities varies widely across the United States, driven by both regional availability of subspecialty care in rheumatology and individual patient factors.

Several studies from different countries have indicated that the rates of orthopedic surgery for patients with RA have decreased over recent years. Recent dramatic improvements in medical treatment might explain the reduction in numbers of orthopedic joint surgeries, which also suggests that there is a worldwide trend toward improved longterm outcomes.

Total joint replacement (TJR) is the most commonly performed RA-related surgical procedure. The rate of TJR performed in patients with RA has either decreased ${ }^{2,3}$ or remained stable ${ }^{4}$. A recent report on administrative discharge databases from 2 large states in the United States has revealed that rates of arthroplasty for patients with RA decreased minimally from 1991 to $2005^{2}$, whereas in Finland, a decline in joint replacement surgery for patients with RA was associated with a concomitant increase in the intensity of antirheumatic therapy from 1995 through $2010^{3}$. Two United Kingdom inception cohorts showed a decline in the rates of hand and foot surgery for patients with RA, but unchanged rates of large joint replacement ${ }^{4}$.

Regardless of these differences in the changing rates of joint replacement surgeries, Nikiphorou, et al have postulated that the unchanged incidence of major interventions, representing mainly surgery to replace large joints, might over time be explained by the preexistence of unrelated osteoarthritis ${ }^{4}$. This is consistent with reports showing increases in the rates of total knee replacement and total hip replacement surgeries in the general population, with a concomitant decrease in TJR in patients with RA.

Our research group recently investigated whether the number of orthopedic operations, including TJR, had decreased among Japanese patients in a single institute-based large observational cohort (Institute of Rheumatology, Rheumatoid Arthritis). We reported that the total number of operations gradually decreased over the period from 2001 to 2007 but increased from 2008 to $2012^{5}$. Regarding TJR, the number of total knee replacement surgeries decreased gradually, while the number of total elbow replacement, total ankle replacement, and artificial finger prosthesis surgeries increased, notably starting in 2008. Moreover, the numbers of finger and foot arthroplasties at our institution have markedly increased. By contrast, Nikiphorou, et al reported that rates of hand and foot surgeries for patients with RA declined from 1986 to 2011 in the United Kingdom ${ }^{4}$. What accounts for the differences between the results of these 2 studies?

Today, disease activity can be successfully controlled in many patients with RA. However, drug therapy cannot perfectly suppress disease activity, and there are many patients who have had RA for a long time before the introduction of treatment with biological disease-modifying antirheumatic drugs. The recent trends in rates of operative procedures may be a reflection of reduced disease activity in patients with longstanding RA who now desire a much better quality of life. With the trend toward milder disease because of improved medical treatment, we speculate that patients with RA may want and seek better function as measured by the activities of daily living. This may also explain the trend toward the increased rate of finger and foot arthroplasties at our institute.
Of course, there are a number of possible explanations for the differences in intervention rates seen in some studies. The data analyzed by our group was from a single institute. Nikiphorou, et al, on the other hand, determined the rates of orthopedic surgeries that were performed over periods as long as a quarter century and recorded in the databases of large multicenter cohorts. Moreover, orthopedic procedures are thought to be influenced by affected joints, the general and local condition of patients, orthopedic surgeons, operative methods, and the healthcare system of each hospital and each country. In the United States, Zhong, et al reported that a significant decline in the rate of arthroplasty and arthrodesis for RA hand and wrist corresponded with increasing density of rheumatologists ${ }^{1}$. Otherwise, tendon reconstruction was not influenced by provider factors, but was correlated with age, race, socioeconomic status, and rural status of the patients ${ }^{1}$.

The decision to undergo surgical intervention naturally depends on a consensus among the patient, orthopedic surgeon, and rheumatologist. In addition, the clinical results obtained for patients with RA depend on the quality of surgical technique. Therefore, we think that "types" of orthopedic surgeries will change in response to changes in drug therapy for RA. Even now, there is a real need to improve patient quality of life, especially for patients with established RA who receive both medical treatment and surgical intervention.

\section{SHIGEKI MOMOHARA, MD, PhD; KOICHIRO YANO, MD; YU}

SAKUMA, MD; KATSUNORI IKARI, MD, PhD, Department of Orthopedic Surgery, Institute of Rheumatology, Tokyo Women's Medical University. Address correspondence to Dr. S. Momohara, Department of Orthopedic Surgery, Institute of Rheumatology, Tokyo Women's Medical University, 10-22 Kawada, Shinjuku, Tokyo, 162-0054, Japan.

E-mail: smomohara@twmu.ac.jp

\section{REFERENCES}

1. Zhong L, Chung KC, Baser O, Fox DA, Yuce H, Waljee JF. Variation in rheumatoid hand and wrist surgery among Medicare beneficiaries: a population-based cohort study. J Rheumatol 2015;42:429-36.

2. Mertelsmann-Voss C, Lyman S, Pan TJ, Goodman SM, Figgie MP, Mandl LA. US trends in rates of arthroplasty for inflammatory arthritis including rheumatoid arthritis, juvenile idiopathic arthritis, and spondyloarthritis. Arthritis Rheumatol 2014;66:1432-9.

3. Jämsen E, Virta LJ, Hakala M, Kauppi MJ, Malmivaara A, Lehto MU. The decline in joint replacement surgery in rheumatoid arthritis is associated with a concomitant increase in the intensity of anti-rheumatic therapy: a nationwide register-based study from 1995 through 2010. Acta Orthop 2013;84:331-7.

4. Nikiphorou E, Carpenter L, Morris S, Macgregor AJ, Dixey J, Kiely $\mathrm{P}$, et al. Hand and foot surgery rates in rheumatoid arthritis have declined from 1986 to 2011, but large-joint replacement rates remain unchanged: results from two UK inception cohorts. Arthritis Rheumatol 2014;66:1081-9.

5. Momohara S, Inoue E, Ikari K, Ochi K, Ishida O, Yano K, et al. Recent trends in orthopedic surgery aiming to improve quality of life for those with rheumatoid arthritis: data from a large observational cohort. J Rheumatol 2014;41:862-6.

J Rheumatol 2016;43:1; doi:10.3899/jrheum.150238 